\title{
Reseña del libro \\ "La Sociedad Postindustrial de Conocimiento: Bases para un análisis del nuevo paradigma educativo"
}

\author{
Eva Andrés Aucejo [1] \\ Universitat de Barcelona. España. \\ eandres@ub.edu \\ "El conocimiento no tiene unidades ni patrones para medir", \\ Zapata-Ros, $M$.
}

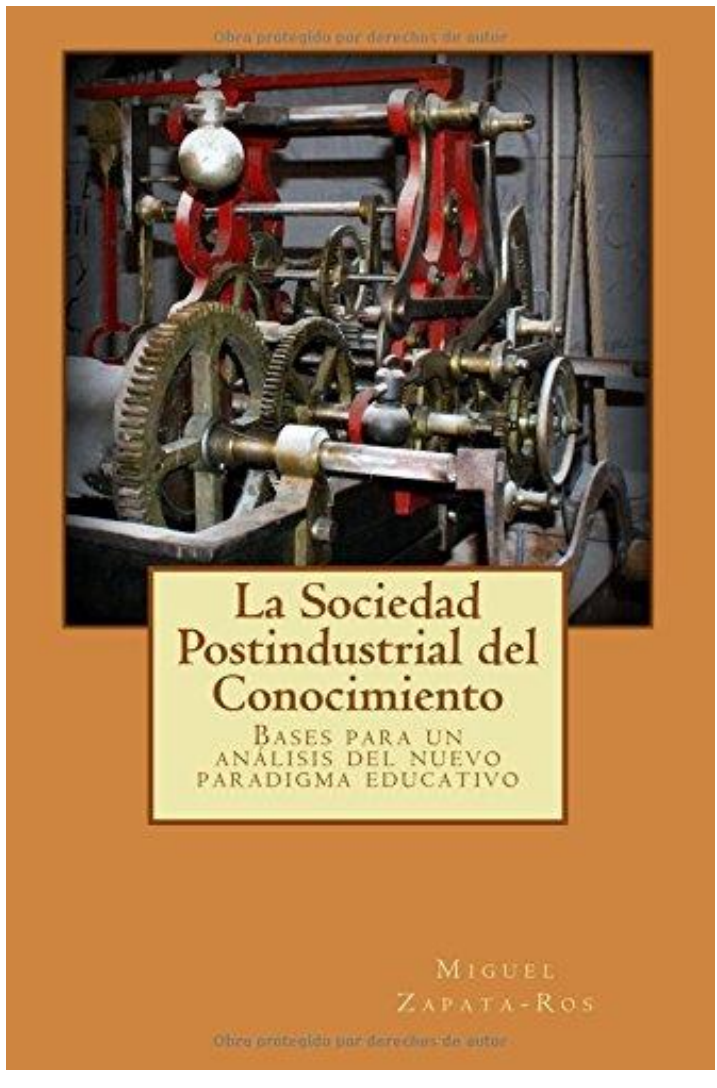

La obra que hoy presento, escrita por el matemático y profesor Miguel Zapata Ros (2015), se conforma de los materiales provenientes de los contenidos de un máster Universitario de Informática interdisciplinar.

Materiales de gran calidad científica que despiertan en el lector un deseo de inmersión en la Sociedad del Conocimiento a través de sus trabadas y bien elaboradas páginas.

Materiales que denotan maestría, selección, método, orden y conocimiento. Conocimiento como origen, trayecto y destino, que culmina en la sociedad postindustrial de Conocimiento por la que, el autor, Miguel Zapata nos conduce con tiento y acierto.

Materiales que transgreden épocas, estilos y disciplinas, cuya puesta en escena nos develan la senda de la Sociedad del Conocimiento.

Materiales con hondos raigambres en la Ciencia de la Filosofía, de la Sociología, de la Economía y otras Ciencias Sociales, que supera su enfoque natural circunscrito a la Ciencia de la Enseñanza y del aprendizaje, conduciendo a un proceso de retroalimentación del propio enfoque didáctico.

Materiales, concluyo, que conforman este gran libro, a cuya lectura invito muy sinceramente. 
La obra referenciada versa sobre la Sociedad Postindustrial del Conocimiento como Sociedad vigente, en la que la información es soportada digitalmente, se procesa masivamente utilizando algoritmos matemáticos y se distribuye a través del gran canal Internet. Resultado, todo ello, en definitiva, del proceso de irrupción de las tecnologías digitales en la Sociedad. Procedimientos y conceptualizaciones de esta nueva Sociedad son descritos por su autor, Zapata-Ros, a través del articulado del libro, en cuyas páginas también reflexiona el autor sobre la naturaleza del conocimiento y de la educación en la Sociedad Postindustrial (como superación de la era industrial y de la ética weberiana del trabajo).

La obra comienza con un primer Capítulo dedicado al concepto de "conocimiento" a cuyo fin se da cita en texto de las más reconocidas y utilizadas conceptualizaciones sobre el tema, emanadas de autores como Platón, pasando por Bertrand Russell, Gettlier, Robert Nozich's, Vittgenstein, Albert, ...

Conceptualizaciones todas ellas ordenadas cronológicamente en el Capítulo II para llegar a la institucionalización del concepto de Sociedad del Conocimiento, versus Sociedad de la Información, gracias a los postulados emanados de las Cumbres Mundiales de la Sociedad de la Información; de la Declaración de Buenos Aires sobre Desarrollo Mundial de las Telecomunicaciones para el Siglo XXI y especialmente de los informes de la UNESCO. Su autor, aprovecha este mismo capítulo segundo para completar una construcción de la idea a través de las notas sobre la Historia de la Teoría de la Sociedad de la Información o del Conocimiento, no olvidando las implicaciones sociales de la nueva Sociedad del conocimiento a través del análisis de términos como burguesía del Conocimiento o Sociedad de propietarios del conocimiento para llegar a abrazar la importantísima noción de "función social del conocimiento".

En el capítulo III se aborda una perspectiva global de la Sociedad del Conocimiento, tratando de alcanzar un concepto integrador que explique las transformaciones sociales, tecnológicas, sociológicas, filosóficas y económicas producidas en la Sociedad Moderna. A tal fin destacan cuatro construcciones epistemológicas de desarrollo conducidas por los siguientes autores. A saber: a) Brurch (2005), quien parte de de la Declaración de Principios de Ginebra adoptada por los Gobiernos y de la Declaración de la Sociedad Civil de la Cumbre Mundial de la Sociedad de la Información y cuya construcción es críticamente analizada por el autor de este libro; b) Nico Stehr (1994) a cuyo juicio la "Sociedad del conocimiento" es la transformación radical y progresiva de la estructura económica de la 'sociedad industrial'; c) Castells (2002) (2001) (1999) (1997), Himanen y Tovald, con un enfoque claramente centrado en la tecnología y d) Evers (2000a, 2000b), quien coordina un grupo de investigación que comparte un enfoque sociológico, entre cuyas aportaciones sin duda destaca la dicotomía en la relación directa que a su juicio se produce entre sociedad desarrollada de conocimiento e ignorancia, resumible en "el saber de lo que no sabemos".

Llegamos así al Capítulo IV dedicado a Conclusiones sobre las características de la Sociedad del Conocimiento. El conocimiento se erige como la principal base de la organización y principal riqueza de la Nueva Sociedad, que ha superado la configuración y características de la Sociedad anterior (industrial).

Reseña del libro "La Sociedad Postindustrial de Conocimiento: Bases para un análisis del nuevo paradigma educativo". Eva Andrés Aucejo.

Página 2 de 4 
En este capítulo el autor se aproxima al concepto a través de un enfoque económico de la Sociedad del Conocimiento que analiza los gastos de las transacciones con bienes del conocimiento y también la gráfica de la progresión de costes en función del volumen de conocimientos o unidades, comparando la sociedad industrial y la Sociedad del Conocimiento. Se repasan también a través de las voces de ilustres autores los rasgos de la sociedad postindustrial estableciendo en unidad de tiempo una reformulación de dichas teorías; se refutan los paradigmas y modelos teóricos que ya no rigen en la actualidad; se ponen de manifiesto las repercusiones más relevantes, acabando este capítulo con un realce del papel y de la importancia del conocimiento "como principal fuente de productividad que ha remplazado a otros factores: Materias primas, trabajo, recursos, información, tecnología o finanzas, en la organización y en la producción".

El autor cierra la obra con dos últimos capítulos: el quinto, dedicado a la Teoría económica sobre el crecimiento de los conocimientos y crecimiento de la ignorancia de Evers (2000), donde el conocimiento y la ignorancia se expresan en sendas funciones exponenciales matemáticas, pero de distintas bases y, finalmente el Capítulo VI dedicado a la Teoría de los Grupos Estratégicos la cual estudia "el origen, el crecimiento y la desintegración de los grupos estratégicos como procesos a largo plazo, así como las configuraciones dinámicas de cooperación y de conflicto entre grupos estratégicos (Evers y Schiel, 1988, pág. 13)”.

\section{Referencias.-}

Burch, S. (2005). Sociedad de la información y sociedad del conocimiento. Palabras en Juego: Enfoques Multiculturales sobre las Sociedades de la Información. Libro coordinado por Alain Ambrosi, Valérie Peugeot y Daniel Pimienta. Editado por C \& F Éditions.

Castells, M. (1997). La era de la información. Economía, sociedad y cultura. Volumen 1, La sociedad red. Alianza Editorial.

Castells, M. (1999). La Era de la Información: Economía, Sociedad y Cultura: La sociedad Red, México, Siglo XXI.

Castells, M. (2001). Internet y la sociedad red. UOC. Lliçó inaugural del programa de doctorat sobre la societat de la informació i el coneixement http://www.uoc.edu/web/cat/articles/castells/print.html

Castells, M. (2002) “La dimensión cultural de Internet”, Universitat Oberta de Catalunya. http://www.uoc.edu/culturaxxi/esp/articles/castells0502/castells0502.html'class='spip_out' rel='nofollow'>http://www.uoc.edu/culturaxxi/esp/a...

Castells, M. (2002). La dimensión cultural de Internet http://www.uoc.edu/culturaxxi/esp/articles/castells0502/castells0502.html

Evers, Hans-Dieter and Solvay Gerke (2005). Knowledge is Power: Experts as Strategic Group.

Stehr, N. (1994). Knowledge Societies. Sage Publications.

Reseña del libro "La Sociedad Postindustrial de Conocimiento: Bases para un análisis del nuevo paradigma educativo". Eva Andrés Aucejo. 
Zapata-Ros, M. (2015). La Sociedad Postindustrial del Conocimiento. Bases para un anàlisis del nuevo paradigma educativo. CreateSpace Independent Publishing Platform; Edición: 1. http://www.amazon.es/Sociedad-Postindustrial-del-Conocimientoparadigma/dp/1519221487/ref=sr_1_4

[1] La Dra. Eva Andrés Aucejo es Catedrática de Derecho Financiero y Tributario, profesora e investigadora de la Universitat de Barcelona. Es Directora de la revista "Education and Law'. La Dra. Andrés es miembro de la Comissió de Postgrau i Formació Continuada de la Facultat de Dret. 